\title{
Aneurysm of the ascending aorta presenting with pulmonary stenosis
}

\author{
M. H. Y ACOUB, M. V. BR A I M B R I D G E, ${ }^{1}$ A N D R. G. G OLD \\ From the Brompton Hospital, London S.W.3
}

The symptoms and signs of aneurysms of the thoracic aorta are due mainly to compression of surrounding mediastinal structures and depend on the size and location of the aneurysm. Compression of the trachea, bronchi, oesophagus, recurrent laryngeal nerve, bone, and superior vena cava is common, but pressure on the pulmonary artery has not been described in spite of its intimate relation to the ascending aorta.

The object of this paper is to describe a case presenting in this way and to discuss the particular surgical problems involved in the excision of such an aneurysm.

\section{CASE REPORT}

E. D., a man aged 52 years, was admitted to the Brompton Hospital in December 1963. He had a history of healed bilateral pulmonary tuberculosis, and he suffered from recurrent attacks of haemoptysis and bronchial infection. In January 1957 he had been admitted to hospital in acute cardiac failure due to coronary thrombosis. Since then he had been moderately dyspnoeic.

On examination he was a heavily built man. The pulse was in sinus rhythm, the blood pressure 120/ $80 \mathrm{~mm}$. $\mathrm{Hg}$, and the jugular venous pressure was normal. An abnormal systolic impulse was palpable in the second left intercostal space at the left sternal edge. There was a moderately loud, harsh, inspiratory pulmonary ejection murmur, and the pulmonary component of the second sound was delayed and reduced in intensity but moved on respiration. Bilateral basal crepitations were heard in the lungs, and the liver was palpable one finger's breadth below the costal margin.

The electrocardiogram showed sinus rhythm with a normal PR interval. The mean frontal $Q R S$ vector was $-40^{\circ}$, and there was right bundle-branch block with a terminal $R^{\prime}$ deflection in $V_{1}$ of $13 \mathrm{~mm}$. These changes were interpreted as indicating ischaemic heart disease.

'Present address: Cardiac Surgical Unit, St. Thomas's Hospital, London S.E.1
The chest radiograph (Fig. 1) showed old bilaterali apical tuberculosis, and both lungs were emphysematous. The emphysema was most marked in the left upper lobe with bullae present. A hemispherical mass 9 9 by $6 \mathrm{~cm}$., lay to the left and in front of the ascend- ing aorta. Its border was slightly irregular. and $\mathbf{3}$ showed flecks of linear calcification laterally and₹ superiorly. The heart size was within normal limits. $\overrightarrow{0}$

At right heart catheterization difficulty was experi-o enced in passing the catheter into the pulmonary artery. The right ventricular systolic pressure waso $44 \mathrm{~mm} . \mathrm{Hg}$ and the pulmonary artery systolic pres sure was $18 \mathrm{~mm}$. $\mathrm{Hg}$, the systolic gradient being $24 \frac{\mathrm{O}}{\mathrm{O}}$ $\mathrm{mm}$. $\mathrm{Hg}$, with a single change in pressure fromarterialo to ventricular configuration in the vicinity of theD pulmonary valve. The angiogram (Fig. 2) showed compression and distortion of the right ventricularo outflow tract, pulmonary valve, and main pulmonary artery by a mass lying above and in front of them The aortic valve and lower part of the ascending aorta were normal. A saccular aneurysm. measuring 8 by $5.5 \mathrm{~cm}$., arose from the upper part of the ascendingo aorta and contained clot. The neck of the sac was $2.5 \mathrm{~cm}$. in diameter. The Wassermann, Price's precipitin reaction, Treponema pallidum immobilization 3 . Reiter protein complement fixation test, cardioliping Wassermann, and fluorescent treponemal antibody 3 tests were all negative.

Repair of the aneurysm was performed on 14 May? 1964 with the aid of profound hypothermia by the Drew technique, using a bilateral transverse incisione. along the lower border of the third rib dividing tho sternum. The circulation was arrested for 35 minutes at a temperature of $12^{\circ} \mathrm{C}$. The aneurysmal sac was opened and the neck was seen to be $2.5 \mathrm{~cm}$. in dia N meter, with strong fibrous margins. Direct suture of the defect in the aortic wall was performed witk interrupted mattress sutures and reinforced with continuous running stitch and a pericardial flap. Two thirds of the sac was removed and the remainder was obliterated by sutures. During rewarming of the patient there was no bleeding from the suture line용

The emphysematous bullae in the left upper lob@ were obliterated by multiple catgut ligatures, a tech $\mathbb{\Omega}$ nique that avoided the air leak which is inevitable with excision and suture. The chest was closed and a tracheostomy was performed. 


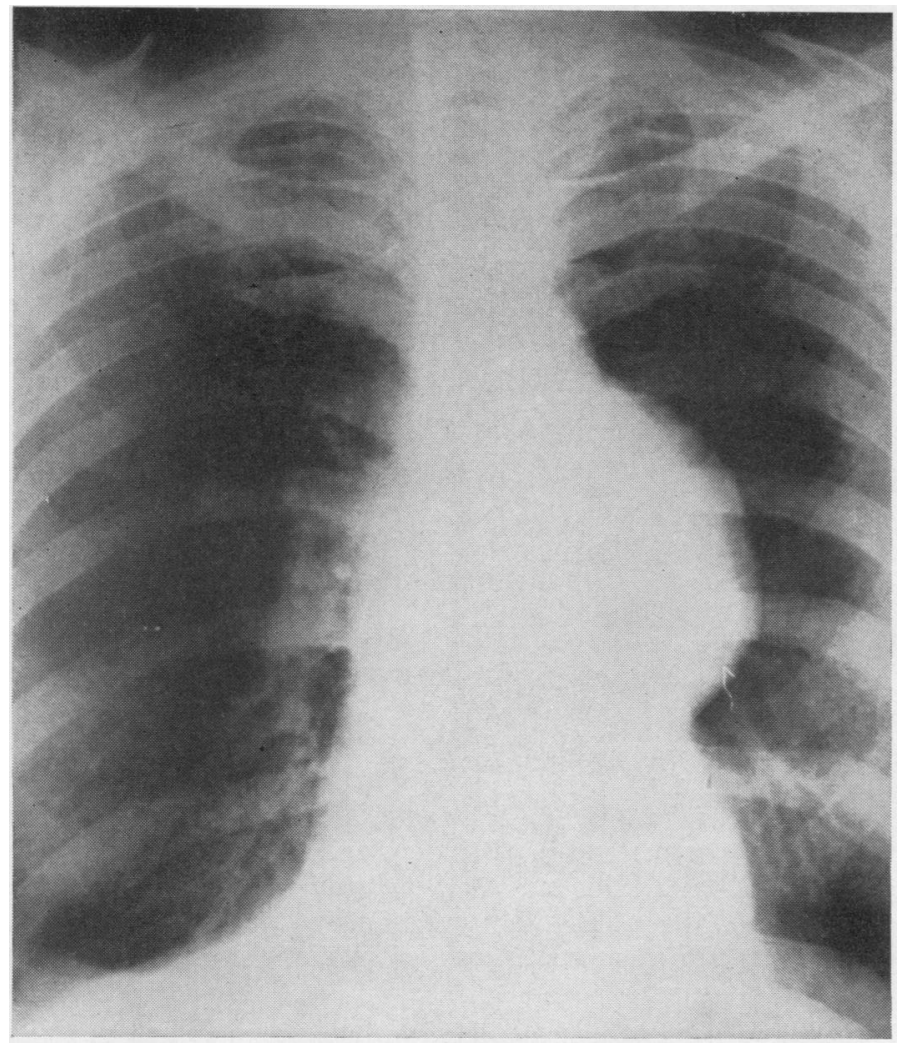

FIG. 1. Pre-operative radiograph shows an ascending aortic aneurysm and healed apical tuberculosis and emphysema.

Artificial ventilation was required for three weeks, following which the patient made an uneventful recovery. The pulmonary ejection murmur disappeared immediately after operation.

When seen in July 1964 he was well, though still somewhat dyspnoeic. On examination the cardiac physical signs were normal except for a blood pressure of $145 / 100 \mathrm{~mm}$. $\mathrm{Hg}$. The electrocardiogram was essentially unchanged from that before operation. His chest radiograph showed a normal aortic outline and diminution of the transverse cardiac diameter (Fig. 3).

\section{DISCUSSION}

Aneurysms of the thoracic aorta may present with signs of compression of the surrounding structures, trachea, bronchi, oesophagus, nerves, bone, and superior vena cava (Boyd, 1924 ; Brindley and Stembridge, 1956 ; Blakemore and Voorhees, 1954 ; Mills and Horton, 1938 ; Kampmeier, 1938). Compression of the pulmonary artery has not been previously described.

Systolic murmurs are frequently observed in patients with aneurysms of the thoracic aorta. Joyce, Fairbairn, Kincaid, and Juergens (1964)

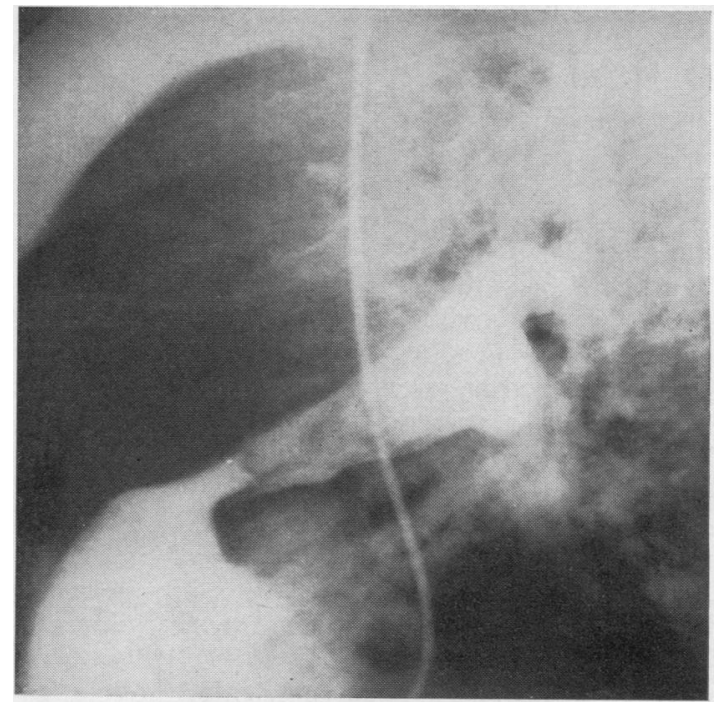

FIG. 2. Lateral angiogram with injection of contrast medium into the right ventricle shows compression and distortion of the right ventricular outflow tract and pulmonary artery. 


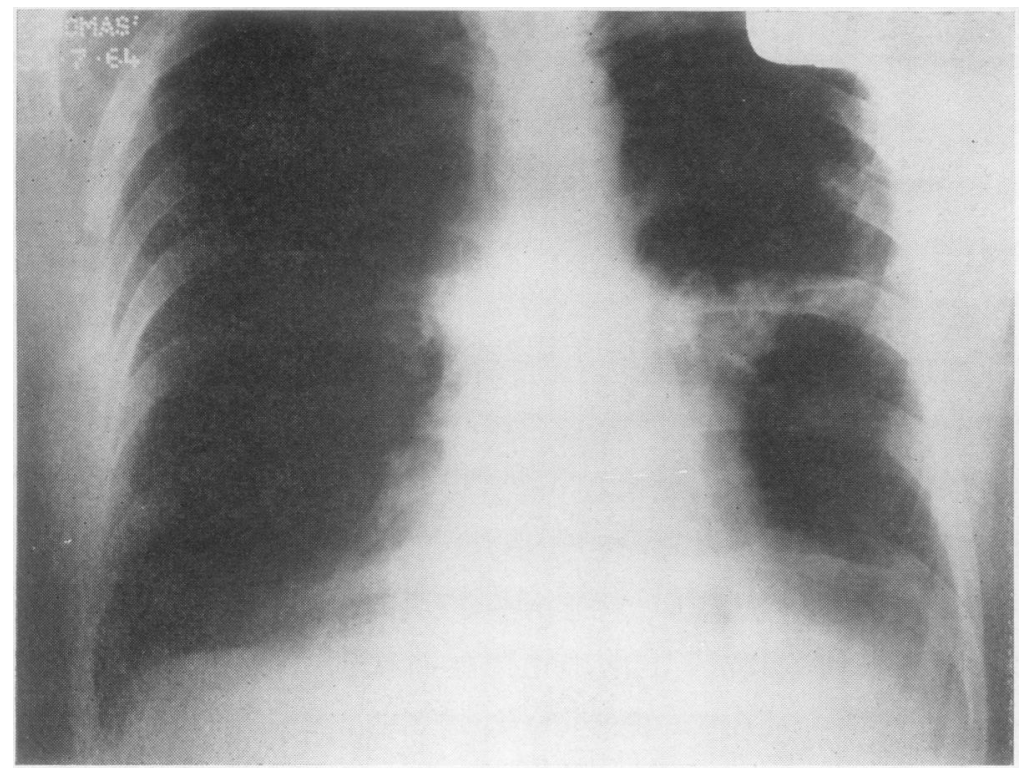

FIG. 3. Post-operative radiograph $\overrightarrow{\mathrm{\omega}}$ shows normal aortic outline and diminution of transverse cardiac $\overrightarrow{\times}$ diameter.

reported that $50 \%$ of a group of 170 patients with aneurysms of the thoracic aorta had murmurs, but concluded that it was difficult to relate them to the aneurysm and that in most patients the murmurs 'seemed to be non-specific'. Mills and Horton (1938) found that 169 out of 339 patients with aneurysm of the thoracic aorta had systolic murmurs which were best heard in the aortic area in 71, at the apex in 52, and in the pulmonary area in 30 patients. In nine patients the systolic murmur was present in all areas and in seven the site of the murmur was not specified.

In the case reported here the systolic ejection murmur was unquestionably pulmonary in origin, as it was louder on inspiration, not conducted to the neck, and was associated with a delayed pulmonary component of the second sound. There was a systolic gradient of $24 \mathrm{~mm}$. $\mathrm{Hg}$ between the right ventricle and the pulmonary artery, and compression of the outflow tract of the right ventricle and main pulmonary artery was confirmed by angiography and at operation. The clinical signs of pulmonary outflow compression were relieved completely by resection of the aneurysm. This suggests that some of the systolic murmurs described in association with aneurysms of the ascending aorta may be due to pulmonary outflow compression.

Obstruction of the right ventricular outflow may therefore be an additional factor in the production of the cardiac enlargement and failure that is common in cases of aneurysm, although these are usually due to associated syphilitic aortic regurgitation, ischaemic heart disease, or systemic hypertension (Brindley and Schwab, 1930 ; Mills and Horton, 1938 ; Brindley and Stembridge, 1956). Relief of pulmonary outflow compression in this patient resulted in diminution of the heart size.

Saccular aneurysms of the ascending aorta compressing the pulmonary outflow present special surgical problems. When the aneurysm has a narrow neck the use of cardiopulmonary bypass has disadvantages. Mobilization and occlusion of the aorta proximal to the aneurysm to allow the coronary arteries to be perfused by the beating 3 . heart is hazardous due to adherence of the aneurysmal sac to the right ventricle and the right coronary and pulmonary arteries. Clamping the 0 aorta distal to the aneurysm necessitates coronary arterial cannulation for myocardial perfusion. This is difficult to do through the narrow neck of the aneurysm and may involve incising normal $\widetilde{N}$ aortic wall above the aortic ring, which is often $N$ obscured by the aneurysm.

These problems do not arise with the use of profound hypothermia by the Drew technique (Drew and Anderson, 1959). With complete circulatory arrest at $12^{\circ} \mathrm{C}$., proximal control and $\cong$ coronary perfusion are unnecessary. The aneurysmal sac is excised, the neck closed from inside, and the redundant sac removed, leaving the part $\stackrel{\mathbb{D}}{\mathbb{D}}$ adherent to the pulmonary and right coronary $\stackrel{\mathcal{D}}{\mathbb{D}}$ arteries.

The main hazard of profound hypothermia in the surgery of thoracic aneurysms is the absence $\delta$ 
of clotting during the rewarming phase, which may cause exsanguinating haemorrhage when plastic prostheses are used because of uncontrollable bleeding though stitch holes in the graft. When the neck is narrow, direct suture of the firm edges of the neck after opening the sac gives a blood-tight suture line, and haemorrhage ceases to be a major problem.

\section{SUMMARY}

A case of aneurysm of the ascending aorta presenting with signs of compression of the pulmonary outflow is described. Resection of the aneurysm resulted in diminution in heart size and improvement of symptoms.

Profound hypothermia of $12^{\circ} \mathrm{C}$. was used to avoid the necessity for mobilizing the aorta and sac, as the latter was adherent to the right ventricle and right coronary and pulmonary arteries.
We would like to thank Mr. N. R. Barrett and Dr. R. V. Gibson for their permission to publish this case.

\section{REFERENCES}

Blakemore, A. H., and Voorhees, A. B., Jr. (1954). Aneurysm of the aorta: a review of 365 cases. Angiology, 5, 209.

Boyd, L. J. (1924). A study of four thousand reported cases of aneurysm of the thoracic aorta. Amer. J. med. Sci., 168, 654.

Brindley, P., and Schwab, E. H. (1930). Aneurysms of the aorta, with a summary of pathologic findings in 100 cases at autopsy. Texas St. J. Med., 25, 757.

- and Stembridge, V. A. (1956). Aneurysms of the aorta: a clinicopathologic study of 369 necropsy cases. Amer. J. Path., 32, 67 .

Drew, C. E., and Anderson, I. M. (1959). Profound hypothermia in cardiac surgery. Lancet, 1, 748 .

Joyce, J. W., Fairbairn, J. F., Kincaid, O. W., and Juergens, J. L. (1964). Aneurysms of the thoracic aorta: a clinical study with special reference to prognosis. Circulation, $29,176$.

Kampmeier, R. H. (1938). Saccular aneurysm of the thoracic aorta: a clinical study of 633 cases. Ann. intern. Med., 12,624 .

Mills, J. H., and Horton, B. T. (1938). Clinical aspects of aneurysm. Arch. intern. Med., 62, 949. 